\title{
Digging into the genome of naked mole-rats
}

Stem-cell research is of great therapeutic potential, as pluripotent stem cells can produce new cells of any adult cell type, and such cells can potentially be used to address many problematic medical conditions. One risk of stem-cell therapy, however, is that injected pluripotent stem cells can produce too many cells without regulation. This can lead to the formation of tumors called teratomas, which can become malignant.

In the context of this concern, Shingo Miyawaki (Hokkaido University) and a team of researchers examined the naked mole-rat, which shows a well-established resistance to cancer (Nat. Commun. 10, $11471 ; 2016)$. The researchers created induced pluripotent stem cells (iPSCs) by reprogramming skin fibroblast tissue from adult mole-rats in order to test its capacity for forming tumors, also called tumorigenicity. They accomplished this by injecting iPSCs into the testes of immune-deficient mice; they also carried out similar injections using iPSCs derived from human tissue and iPSCs from mouse tissue. As expected, these latter injected iPSCs produced teratomas in the testes of immune-deficient mice; however, iPSCs from mole-rats did not.

To establish potential explanations for this tumor resistance, Miyawaki's team compared gene expression in mole-rat iPSCs to that of mole-rat fibroblasts and iPSCs derived from humans and mice. They identified that a tumor-suppressor gene, $A R F$, is highly expressed in mole-rat iPSCs but suppressed in human- and mousederived iPSCs. Additionally, mole-rat iPSCs have a disrupted ERAS gene, which is expressed in human- and mouse-derived iPSCs and is associated with tumorigenesis.

Armed with these putative causes, the researchers modulated these genes in the different types of iPSCs to observe how $A R F$ and ERAS mediate tumorigenesis. In mole-rat iPSCs, the researchers disrupted $A R F$ with targeted short hairpin RNAs and added the ERAS gene from mice; the resulting iPSCs, when injected, produced large tumors. Conversely, by forcing the expression of the Arf gene in mouse-derived iPSCs, the researchers achieved lower tumorigenicity after injection.

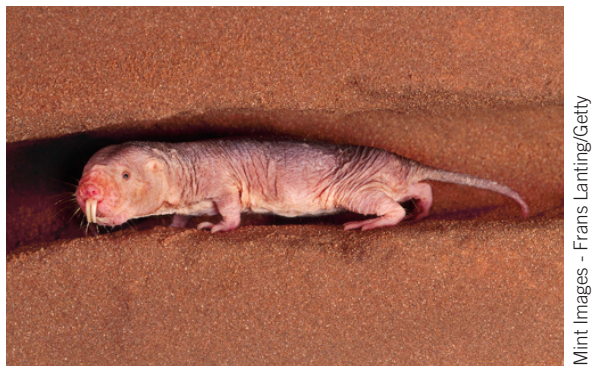

These findings provide convincing evidence that $A R F$ and ERAS significantly affect the development of teratomas from iPSCs. In their manuscript, the authors note this potential direction for biomedical discovery, suggesting that further research into the mechanisms by which $A R F$ suppresses tumors "may contribute to the generation of non-tumorigenic human-iPSCs enabling safer cell-based therapeutics." On its own, however, this study demonstrates the value of the naked mole-rat as a model for cancer resistance, with work already underway to demystify and translate its unique resilience into biomedical applications.

Gregory D. Larsen

\section{PROMISING VACCINES FOR ZIKA}

The Zika virus (ZIKV) continues to loom in the international sphere of world health, and researchers are exploring potential strategies for treatment and prevention of the disease, even as they seek a better understanding of its effects on prenatal development, which include fetal microcephaly, restriction of intrauterine growth and other birth defects in both humans and mice. In response to concerns about the continuing spread and devastating consequences of ZIKV, many regulatory bodies and research groups have accelerated development of a vaccine for the virus. Now, in an 'accelerated article preview', Nature has published a research letter from scientists of Walter Reed Army Institute of Research (Silver Spring, MD), University of São Paulo (Brazil), the Beth Israel Deaconess Medical Center (Boston, MA) and Harvard Medical School (Cambridge, MA) that describes the early fruits of this accelerated effort.

This team, led by senior author Dan Barouch, used Balb/c mice in preclinical challenge tests to assess the efficacy of two vaccines: a DNA vaccine and a purified inactivated virus vaccine (Nature doi:10.1038/nature18952; published online 28 June 2016). The DNA vaccine works by introducing custom-designed plasmids that encode for antigens of known proteins in ZIKV; once injected, these plasmids are taken up by nearby cells, which thereafter directly express the engineered antigen and induce a corresponding immunological response. By contrast, the inactivated virus vaccine was developed by purifying ZIKV from inoculated cells and inactivating the virus with formalin; once injected, the inactivated viral particles themselves induce an immunological response without causing infection. Notably, both of these platforms have been used previously to develop vaccines for other viruses of ZIKV's genus, called flaviviruses.

When mice were infected intravenously with active viral particles, those that had received a single immunization with the DNA vaccine were completely protected against the ZIKV challenge, whereas sham-vaccinated controls showed 6 days of detectable viremia. Similarly, mice that received an intramuscular injection of the purified inactivated virus showed complete protection during a ZIKV challenge, although this vaccine was less effective when administered by subcutaneous injection.

The authors prudently note that such success in a mouse model cannot be interpreted to forecast success with humans, but this remains an indisputably positive advancement toward the development of a ZIKV vaccine. In a press release, Barouch acknowledged the encouraging results of his team's work, noting, "We hope that this news will electrify and galvanize the vaccine effort against Zika virus." 